\title{
Características cineantropométricas e ingesta nutricional de mujeres y hombres costarricenses dedicados al modelajepublicitario Kinanthropometric characteristics and nutritional intake of Costa Rican female and maleadvertising models

\author{
Yamileth Chacón-Araya, Catalina Fernández-Campos, José Moncada-Jiménez \\ *Universidad de Costa Rica (Costa Rica), **Comité Olímpico de Costa Rica (Costa Rica), ***Centro de Investigación en Ciencias del \\ Movimiento Humano (Costa Rica)
}

\begin{abstract}
Resumen. El propósito del estudio fue describir las características cineantropométricas y la ingesta nutricional de mujeres y hombres costarricenses dedicados al modelaje publicitario. Se realizó un estudio transversal descriptivo con modelos y controles. Se midieron características cineantropométricas y la ingesta de alimentos. Participaron 135 personas, divididas en grupos de modelos (mujeres, $\mathrm{n}=35$, hombres, $\mathrm{n}=18$ ) $\mathrm{y}$ controles (mujeres, $\mathrm{n}=40$, hombres, $n=42$ ). Independientemente del sexo, el porcentaje de grasa corporal fue menor en las personas que se dedican al modelaje que los participantes control $(p<.001)$. Las mujeres modelos tenían un menor índice de conicidad que las mujeres controles $(p<.001)$, y los hombres modelos y los controles tuvieron un índice de conicidad similar $(p=.692)$. El arroz y los frijoles fueron los carbohidratos complejos más comunes en la dieta de los sujetos en general. En comparación con los controles, los modelos presentan una menor frecuencia de consumo de arroz, un mayor consumo de verduras harinosas y cereales integrales, una preferencia por grasas saludables y menor consumo de galletas dulces, repostería y aceite vegetal. En conclusión, las personas que se dedican al modelaje tienen una adiposidad menor e ingieren alimentos en porciones más saludables que quienes no son modelos.

Palabras claves: modelaje, Costa Rica, alimentación, nutrición, cineantropometría.
\end{abstract}

Abstract. The purpose of the study was to describe the kinanthropometric characteristics and nutritional intake of female and male Costa Rican advertising models. Models and controls participated in a cross-sectional study. Kinanthropometric characteristics and food intake were measured. This is a cross-sectional study in which models and controls completed questionnaires. Kinanthropometric characteristics and food intake were measured. Participants were 135 subjects divided into groups of models (females, $n=35$, males, $n=18$ ) and controls (females, $n=40$, males, $n=42$ ). Regardless of gender, body fat percentage was lower in models than in control participants $(p<.001)$. Female models had a lower conicity index than female controls $(p<.001)$, and male models and controls had a similar conicity index $(p=.692)$. Rice and beans were the most common complex carbohydrates in the diet of individuals in general. Compared to controls, the models had a lower frequency consumption of rice, higher starch vegetables and whole grains intake, a preference for healthy fats and lower intake of sweet cookies, pastries and vegetable oil. In conclusion, advertising models had a lower adiposity and their food consumption consisted on healthier portions than their control counterparts.

Key words: models, Costa Rica, food intake, nutrition, kinanthropometry.

\section{Introducción}

La salud de las personas que se dedican al modelaje profesional ha recibido escasa atención, especialmente en mercados de moda emergentes como Costa Rica (Moncada-Jiménez, 2006). En el ámbito internacional, se ha puesto especial atención a las características físicas que pueden estar asociadas a problemas de salud, como por ejemplo, un bajo peso corporal para la estatura de las mujeres o desórdenes de alimentación (Santonastaso, Mondini, \& Favaro, 2002).

Una parte de la literatura relacionada con este tema ha descrito las características físicas de modelos que aparecen en las revistas de moda, y su énfasis se ha centrado en las mujeres (Morris, Cooper, \& Cooper, 1989). Morris et al. (1989), analizaron las mujeres de una agencia de modelaje de los años 1967 a 1987, encontrando un aumento significativo en la estatura y una reducción significativa en la cintura de las mujeres, así como una tendencia al aumento en el perímetro mesoesternal (i.e., tamaño del busto, mamas, senos). No se encontraron cambios en el diámetro de la cadera. Con los años el cambio de la figura de las mujeres, va de una mujer con más curvas a una con forma más tubular o cilíndrica. Esta característica se puede evaluar con el índice de conicidad, el cual representa la figura humana como un cilindro; es decir, las personas con menor volumen en el área abdominal serían consideradas como un cilindro perfecto o lo que se conoce como perfil andrógeno (Valdez, Seidell, Ahn, \& Weiss, 1993).

También se han realizado estudios (Sypeck, Gray, \& Ahrens, 2004), en los que se ha analizado el cuerpo ideal o la figura ideal femenina de mujeres estadounidenses desde 1959 hasta 1999. Sin embargo, en este estudio se revisaron únicamente revistas de moda, en donde los investigadores encontraron que el tamaño general de las modelos se redujo

Fecha recepción: 21-03-16. Fecha de aceptación: 07-06-16

Yamileth Chacón-Araya

yamileth.chacon@ucr.ac.cr significativamente en el periodo comprendido entre 1980 y 1990, así como un aumento en la frecuencia en que las revistas presentaban a la mujer de cuerpo entero de 1960 a 1990. Los investigadores concluyen que la sociedad americana, específicamente la estadounidense, valora mucho el ideal de mujer muy delgada y que se presenta de cuerpo entero en una publicación, lo cual refuerza el estereotipo de mujer ideal que se exporta a otros países que reciben una influencia importante de la publicidad de los Estados Unidos de América (Sypeck et al., 2004).

También se ha estudiado, aunque escasamente, la prevalencia de desórdenes de alimentación en modelos femeninas (Santonastaso et al., 2002). En ese estudio también se investigó el uso de drogas ilícitas, y se encontró que el de las modelos era mayor que el de las mujeres que no eran modelos. Para llegar a esa conclusión, los investigadores estudiaron a 63 modelos profesionales y las compararon con 126 mujeres de la población general, y encontraron que las modelos tenían un peso corporal significativamente menor que las mujeres de la población general. En cuanto al tema de los desórdenes de alimentación encontraron diferencias significativas entre ambos grupos de mujeres. El uso de sustancias estimulantes o de alcohol entre las modelos fue del 35\% y el de las mujeres de la población general fue del $12 \%$. Los investigadores concluyeron que las modelos poseen un mayor riesgo de tener desórdenes de alimentación y el uso de drogas ilícitas que las mujeres de la población general. Se han encontrado hallazgos similares respecto a problemas de alimentación en modelos italianas (Preti, Usai, Miotto, Petretto, \& Masala, 2008), en donde además se ha reportado que un $54.5 \%$ de las modelos poseen un índice de masa corporal (IMC = peso en kg/estatura en $\mathrm{m}^{2}$ ) menor de $18 \mathrm{~kg} / \mathrm{m}^{2}$; valor considerado como de desnutrición o un peso muy bajo para la estatura de esa persona (American College of Sports Medicine, 2010, 2014).

En este contexto, actualmente se sabe acerca de las características físicas y algunos patrones de alimentación de modelos estadounidenses y europeas, los cuales evidentemente pueden afectar negativamente su salud. Sin embargo, no se conocen los hábitos de alimentación ni las características físicas de modelos latinoamericanas, y mucho menos, de modelos masculinos. Por lo tanto, el propósito del estudio fue describir 
las características físicas y la ingesta nutricional en mujeres y hombres que se dedican al modelaje publicitario en Costa Rica.

\section{Metodología}

\section{Diseño del estudio}

El diseño del estudio fue descriptivo transversal (Montero \& León, 2002). Los participantes fueron medidos solamente una vez y se comparan los grupos de interés con un grupo control del mismo sexo y edad.

\section{Participantes}

Se reclutaron mujeres y hombres que trabajaban en modelaje, así como mujeres y hombres que servirían para comparación (i.e., controles). Estas personas fueron invitadas a participar reclutándolas de agencias de modelaje, a través de anuncios en redes sociales (e.g., Facebook), una noticia periodística y por referencia de los mismos participantes. En total, se reclutaron 135 personas, las cuales fueron divididas en grupos de modelos (mujeres, $\mathrm{n}=35$, hombres, $\mathrm{n}=18$ ) y controles (mujeres, $n=40$, hombres, $n=42$ ).

\section{Criterios de inclusión y exclusión}

Las y los modelos se reclutaron si trabajaban profesionalmente o semi profesionalmente (i.e., tiempo parcial) en modelaje. Se definió que se reclutaría modelos que participaban en medios de comunicación(e.g., T.V., prensa, revistas) o que fueran contratadas para eventos de modelaje en vivo (e.g., pasarelas, acompañamiento a eventos). Se definió reclutar participantes entre 18 y 35 años de edad. Se excluyeron del análisis los participantes que no completaran los instrumentos de medición.

\section{Instrumentos y procedimientos de medición}

Las personas interesadas en participar el estudio fueron citadas al Centro de Investigación en Ciencias del Movimiento Humano (CIMOHU) de la Universidad de Costa Rica. Allí se les recibió y se les explicó el procedimiento a seguir, comenzando por el consentimiento informado para participar como sujeto en una investigación aprobado por el Comité Ético Científico de la Universidad de Costa Rica. Posteriormente, se les solicitó el nombre completo y la fecha de nacimiento.

Para la medición de la estatura corporal en cm se utilizó un estadiómetro metálico fijo (Novel Products Inc., modelo DES 290-337, Rockton, USA) con una precisión de $.5 \mathrm{~cm}$. El peso corporal en $\mathrm{kg}$ se midió con una balanza electrónica marca Tanita (modelo BF-682 W, Japón), con una precisión de .1 g. Con los valores de la talla y el peso corporal se calculó el IMC. Los valores normales del IMC (18.5-24.9 $\mathrm{kg} / \mathrm{m}^{2}$ ) y la cintura, tanto para mujeres $(<88 \mathrm{~cm})$ como para hombres $(<$ $102 \mathrm{~cm}$ ) fueron interpretados de acuerdo a valores de referencia internacionales (American College of Sports Medicine, 2014).

Para medir la estatura, primero se ubicó al participante en el estadiómetro, descalzo, con la cabeza, espalda, glúteos y piernas apoyadas en la pared y con los pies a $60^{\circ}$. Después se le pidió inspirar profundamente y se anotó la estatura en cm (American College of Sports Medicine, 2010, 2014). Luego, se le solicitó a la persona subir a la báscula, utilizando la menor cantidad de ropa posible y sin accesorios como reloj o teléfono celular. Una vez que la persona subió a la báscula se le solicitó no moverse y luego se leyó el peso en kg. Posteriormente, cada participante completó un instrumento para conocer factores demográficos, tipos de modelaje en el que participaban, y el instrumento para conocer la ingesta nutricional.

La composición corporal (i.e., porcentaje de grasa corporal, densidad mineral ósea [DMO]) se obtuvo por medio de absorciometría de energía dual de rayos X (DXA), con un densitómetro marca General Electric (modelo Lunar Prodigy Advance, Madison, WI, USA) con la versión de software enCore 2011, \# 13,60,033. Este instrumento es considerado el estándar de oro para la determinación de la DMO, la cual se obtiene en unidades de $\mathrm{g} / \mathrm{cm}^{2}$ y posee un error de medición de $<2 \%$ (Carbuhn, Fernandez, Bragg, Green, \& Crouse, 2010; Ito et al., 2001; Nana, Slater, Stewart, \& Burke, 2015; Valdimarsson, Alborg, Duppe, Nyquist, \& Karlsson, 2005). A la persona se le preguntó si poseía alguna fractura o algún objeto metálico en el cuerpo. Si el objeto metálico podía ser retirado de su cuerpo (e.g., anillos, pulseras u otros accesorios), se le indicó que lo hiciera. Si no lo podía remover de su cuerpo, se llevaba a cabo un proceso de depuración por medio del software del equipo DXA, de manera que el dato adquirido fuera válido. Alas mujeres se les preguntó si estaban embarazadas o no (no hubo participantes embarazadas). Posteriormente, se les pidió ubicarse de cúbito dorsal en el equipo de densitometría (i.e., DXA), para prepararlas para medir la composición corporal. Una vez realizado el estudio correspondiente, se les pidió incorporarse lentamente. El tamaño del busto (solo en mujeres), y las circunferencias de cintura y cadera fueron medidas con una cinta Gulick (Creative Health Products, Ann Arbor, MI, USA). Se dividió la circunferencia de la cintura entre la de cadera para obtener la relación cintura/cadera, y los valores del porcentaje de grasa para cada sexo fueron interpretados de acuerdo con las normas internacionales (American College of Sports Medicine, 2010, 2014).

El índice de conicidad se calculó para evaluar el grado de adiposidad abdominal y la forma corporal con la siguiente fórmula: circunferencia de cintura / (.109 x «(peso corporal /estatura)) (Valdez et al., 1993). La circunferencia de la cintura y la estatura se expresan en metros y el peso en kilogramos, el valor .109 es una constante que resulta de convertir unidades de volumen y masa en unidades de longitud. El índice de conicidad no tiene unidades propias y su rango varía entre 1 que representaría un cilindro perfecto y 1.73 que es indicativo de un doble cono unido por la base.

Para medir la ingesta nutricional se aplicó un cuestionario de frecuencia de consumo de alimentos utilizado previamente en Costa Rica (Fernandez-Campos, Dengo, \& Moncada-Jimenez, 2015). El cuestionario consiste de una lista de 47 alimentos, de los cuales el participante debe indicar la frecuencia con que los consume: 1) todos los días, 2) 3 a 4 veces por semana, 3) 1 a 2 veces por semana, y 4) nunca. Con la información proporcionada por cada persona, se determina la distribución(\%) de macronutrientes según componente mayoritario. Para obtener esta información, se analizan los ítems 1 al 21 para obtener la distribución de carbohidratos, los ítems 22 al 33 para las proteínas, y los ítems 34 al 47 para las grasas (Fernandez-Campos et al., 2015).

\section{Análisis estadístico}

Para el análisis de los datos recolectados se utilizó el Paquete Estadístico para las Ciencias Sociales (SPSS, IBM Corporation, Armonk, New York, USA), versión 20.0. Se obtuvieron estadísticas descriptivas media (SD) para las variables continuas: edad, peso, estatura, IMC, circunferencia de cintura, cadera, tamaño del busto, relación cintura/ cadera, índice de conicidad, porcentaje de grasa corporal, y densidad mineral ósea. Se presentan porcentajes para describir las variables categóricas de alimentación.

El análisis inferencial incluyó pruebas t student de grupos independientes para comparar la circunferencia del busto en mujeres modelos y controles. Se utilizaron pruebas de análisis de varianza(ANOVA) factorial para 2 grupos (modelos, controles) y 2 sexos (mujeres, hombres) en la variables edad, peso, estatura, IMC, circunferencia de cintura, cadera, tamaño del busto, relación cintura/cadera, índice de conicidad, porcentaje de grasa corporal, y densidad mineral ósea. Finalmente, se utilizaron correlaciones producto momento de Pearson para estudiar la asociación entre las variables continuas. Todos los análisis se consideraron estadísticamente significativos si alcanzaban una $p<.05$.

\section{Resultados}

En la tabla 1 se describen las características de los participantes. La experiencia en modelaje de las mujeres era de 5.16(4.13) años, mientras que la de los hombres era de 4.11 (3.67) años. Todos los participantes completaron la educación primaria y más del $77 \%$ estaba cursando educación universitaria o ya había finalizado alguna carrera universitaria.

El tipo de modelaje indicado por los participantes fue diverso (Tabla 2). Cabe anotar que las y los modelos podrían seleccionar más de una opción. En las mujeres, destacan entre los tres primeros lugares, el 
Tabla 1.

Escolaridad de los participantes en el estudio (los valores representan \%).

\begin{tabular}{lcccc}
\hline & \multicolumn{2}{c}{ Mujeres $(\mathbf{n}=\mathbf{7 5})$} & \multicolumn{2}{c}{ Hombres $(\mathbf{n}=\mathbf{6 0})$} \\
\cline { 2 - 5 } Escolaridad & $\begin{array}{c}\text { Modelo } \\
(\mathbf{n = 3 5})\end{array}$ & $\begin{array}{c}\text { Control } \\
(\mathbf{n}=\mathbf{4 0})\end{array}$ & $\begin{array}{c}\text { Modelo } \\
(\mathbf{n}=\mathbf{1 8})\end{array}$ & $\begin{array}{c}\text { Control } \\
(\mathbf{n}=\mathbf{4 2})\end{array}$ \\
\hline Secundaria completa & 11.4 & 2.6 & 16.7 & 7.1 \\
Secundaria incompleta & 11.4 & 2.6 & 5.6 & 2.4 \\
Universitaria completa & 25.7 & 20.5 & 22.2 & 16.7 \\
Universitaria incompleta & 51.4 & 74.4 & 55.6 & 73.8 \\
\hline
\end{tabular}

Tabla 2.

Tipo de modelaje en el que trabajaron las y los modelos del estudio $(n=53)$.

Tipo de modelaje

Artístico

Pintura del cuerpo ("Body Paint”)

Anuncios comerciales

Edecán

Eventos

Fitness o acondicionamiento físico

Fotografía

Maniquí

Pasarela

Presentadora T.V.

Promotora

Revistas

Ropa interior

Televisión

Traje de baño Mujeres ( $\mathbf{n}=\mathbf{3 5})$

\section{3}

14.3
2.9
2.9

2.9
85.7

85.7
20.0
80.0

5.7

65.7
2.9

2.9
2.9

0

11.4

68.6
42.9

Nota: Los valores representan el porcentaje de respuestas en cada categoría.

Tabla 3.

Estadísticas descriptivas de los participantes $(\mathrm{n}=135)$.

\begin{tabular}{|c|c|c|c|c|}
\hline \multirow[b]{2}{*}{ Variable } & \multicolumn{2}{|c|}{ Mujeres $(n=75)$} & \multicolumn{2}{|c|}{ Hombres $(n=60)$} \\
\hline & $\begin{array}{r}\text { Modelos } \\
(\mathrm{n}=35)\end{array}$ & $\begin{array}{l}\text { Control } \\
(\mathrm{n}=40)\end{array}$ & $\begin{array}{r}\text { Modelos } \\
(\mathrm{n}=18)\end{array}$ & $\begin{array}{l}\text { Control } \\
(\mathrm{n}=42)\end{array}$ \\
\hline Edad (años) & $23.46(5.15)$ & $21.50(3.20)$ & $24.83(4.74)$ & $23.02(3.32)$ \\
\hline Estatura (cm) & $163.31(5.00)$ & $159.96(7.03)$ & $177.54(6.42)$ & $173.33(6.89)$ \\
\hline Peso $(\mathrm{kg})$ & $55.43(4.84)$ & $58.13(9.09)$ & $75.40(8.78)$ & $71.23(9.29)$ \\
\hline $\begin{array}{l}\text { Índice de masa corporal } \\
\left(\mathrm{kg} / \mathrm{m}^{2}\right)\end{array}$ & $20.79(1.65)$ & $22.67(2.86)$ & $23.92(2.45)$ & $23.66(2.31)$ \\
\hline Cintura $(\mathrm{cm})$ & $62.25(3.66)$ & $66.81(6.15)$ & 76.64 (3.93) & 75.66 (5.79) \\
\hline Cadera (cm) & $91.38(3.91)$ & $93.71(6.75)$ & $95.44(4.65)$ & $93.89(6.02)$ \\
\hline Relación cintura/cadera & $0.68(0.04)$ & $0.71(0.04)$ & $0.80(0.02)$ & $0.81(0.04)$ \\
\hline Masa grasa (\% DXA) & 28.55 (6.79) & $34.22(7.02)$ & $14.85(6.51)$ & $18.18(6.32)$ \\
\hline $\begin{array}{l}\text { Densidad mineral ósea } \\
\left.\text { cuerpo entero (g/ } \mathrm{cm}^{2}\right)\end{array}$ & $1.13(0.10)$ & $1.11(0.07)$ & $1.23(0.08)$ & $1.22(0.08)$ \\
\hline Índice de conicidad & $0.98(0.04)$ & $1.02(0.05)$ & $1.08(0.03)$ & $1.09(0.05)$ \\
\hline
\end{tabular}

modelaje en eventos especiales, la fotografía y apariciones en la televisión; mientras que en los hombres son más frecuentes los trabajos en fotografía, pasarela y eventos especiales. Aunque en Costa Rica se han observado mujeres en el modelaje de pintura sobre el cuerpo o «body paint», ninguna de las participantes de este estudio indicó haberlo hecho, aunque sí lo hizo un hombre.

En la tabla 3 se presenta la estadística descriptiva de los participantes $(n=135)$. El IMC promedio de todos los participantes se encuentra dentro del rango considerado como normal (18.5-24.9 kg/m²), así como los valores de cintura, tanto para mujeres $(<88 \mathrm{~cm})$ como para hombres (<102 cm) (American College of Sports Medicine, 2014). Sin embargo, $14.3 \%$ de las mujeres modelos y $2.5 \%$ de las mujeres controles se encontraban por debajo de $18.5 \mathrm{~kg} / \mathrm{m}^{2}$, lo cual las clasifica como personas con bajo peso para su estatura. Por medio del DXA, se encontraron tres modelos mujeres con escoliosis, sin que este llegara a ser un hallazgo estadísticamente significativo.

Los valores de la relación cintura/cadera se encuentran dentro de lo considerado normal para hombres $(<0.90)$ y para mujeres $(<0.85)$ (American College of Sports Medicine, 2014). En cuanto al porcentaje de grasa corporal, los valores para las mujeres modelos están dentro del promedio(25-31\%)(American College of Sports Medicine, 2010, 2014), pero el de las mujeres control se encuentra sobre el promedio (Tabla 3). Cabe mencionar que $31.4 \%$ de las mujeres modelos y el $12.5 \%$ de las controles presentaron porcentaje de grasa corporal inferiores a 25\%. En el caso de los hombres modelos, los valores están dentro de lo considerado como bajo el promedio (18-24\%), y el de los hombres controles se encuentran dentro del valor normal (Tabla 3) (American College of Sports Medicine, 2014). Cabe mencionar que 72.2\% de los hombres modelos y el $50.0 \%$ de las controles presentaron porcentaje de grasa corporal inferiores a $18 \%$.

Las pruebas de ANOVA 2 x 2 (grupos x sexo) no revelaron interacciones significativas en las variables de edad $(p=.920)$, estatura $(p=.714)$ circunferencia de la cadera $(p=.062)$, porcentaje de grasa corporal $(p=.338)$, y DMO $(p=.673)$. Sin embargo, en estas variables si se encontraron efectos principales estadísticamente significativos. En el caso de la edad, se encontró que independientemente del sexo, las personas que se dedican al modelaje tenían una edad mayor (media = 24.15 (.59) años) que los participantes control (media = 22.26 (.45) años) $(p=.012)$. En el caso de la estatura corporal se encontró que independientemente del sexo, las personas que se dedican al modelaje tenían una estatura mayor (media $=170.43(.93) \mathrm{cm}$ ) que los participantes control (media $=166.64(.71) \mathrm{cm})(p=.002)$. Además, independientemente del grupo, los hombres presentaron una mayor estatura (media $=175.43(.91) \mathrm{cm}$ ) que las mujeres (media $=161.64(.75) \mathrm{cm})(p<.001)$. De la misma forma, los hombres presentaron una mayor circunferencia de la cadera $($ media $=94.67(.80) \mathrm{cm}$ ) que las mujeres $($ media $=92.54$ $(.65) \mathrm{cm})(p=.041)$. Independientemente del sexo, el porcentaje de grasa corporal medido con DXA, fue significativamente menor en las personas que se dedican al modelaje (media = 21.70 (.97) \%) que los participantes control (media $=26.20(.74) \%)(p d » .001)$. Por otro lado, independientemente del grupo, los hombres tuvieron un menor porcentaje de grasa corporal (media $=16.51(.94) \%$ ) que las mujeres (media = 31.39 (.77) \%). La DMO, independientemente del sexo, fue mayor en las personas que se dedican al modelaje (media $\left.=1.18(.12) \mathrm{g} / \mathrm{cm}^{2}\right)$ que la de los participantes control (media $\left.=1.17(.01) \mathrm{g} / \mathrm{cm}^{2}\right)(p<.001)$.

Las pruebas deANOVA $2 \times 2$ (grupos $x$ sexo) revelaron interacciones significativas en las variables de peso corporal ( $p=.024)$, IMC ( $p=$ $.014)$, circunferencia de la cintura $(p=.004)$, relación cintura/cadera ( $p=$ .033), e índice de conicidad ( $p=.035$ ). Los análisis de las interacciones indicaron, en el caso del peso, que los hombres modelos tenían un mayor peso que las mujeres modelos $(p<.001)$ y que los hombres controles tenían un mayor peso que las mujeres controles ( $p \mathrm{~d}$ » .001) (Tabla 3). En el caso del IMC, se encontró que las mujeres modelos tenían un menor IMC que las mujeres controles $(p<.001)$. Los hombres modelos y los controles tuvieron IMC similares $(p=.696)$ (Tabla

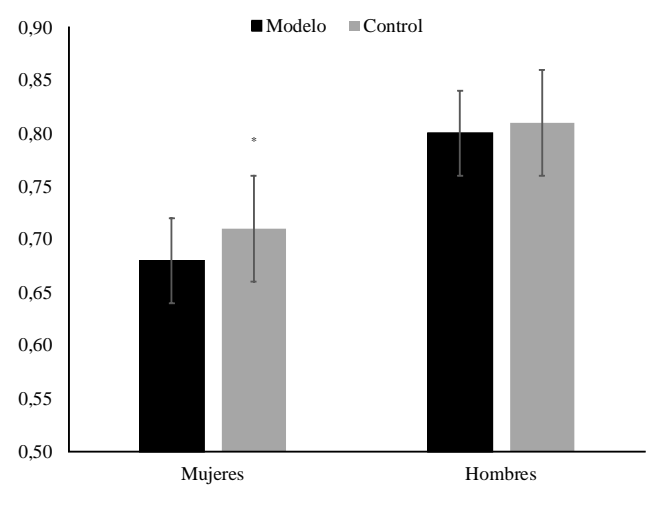

Figura 1. Relación cintura/cadera en mujeres y mujeres

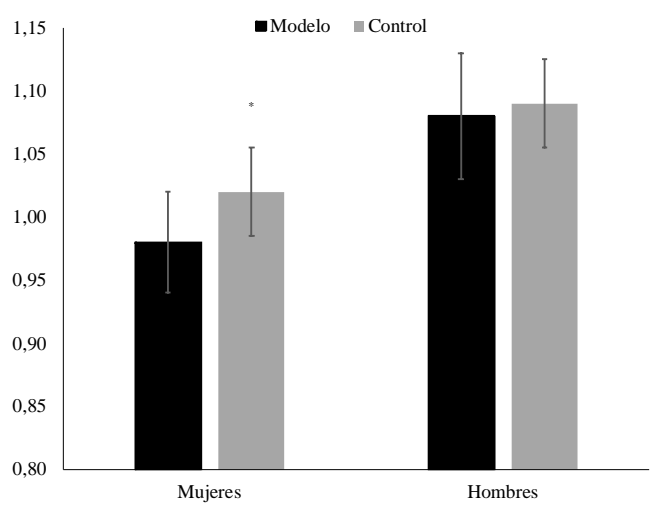

Figura 2. Índice de conicidad en mujeres y hombres. 
Tabla 4 .

Consumo diario de carbohidratos reportado por los participantes.

\begin{tabular}{lcccc}
\hline & \multicolumn{2}{c}{ Mujeres $(\mathbf{n}=\mathbf{7 5})$} & \multicolumn{2}{c}{ Hombres $(\mathbf{n}=\mathbf{6 0})$} \\
\cline { 2 - 5 } Consumo diario & $\begin{array}{c}\text { Modelo } \\
(\mathbf{n}=\mathbf{3 5})\end{array}$ & $\begin{array}{c}\text { Control } \\
(\mathbf{n}=\mathbf{4 0})\end{array}$ & $\begin{array}{c}\text { Modelo } \\
(\mathbf{n}=\mathbf{1 8})\end{array}$ & $\begin{array}{c}\text { Control } \\
(\mathbf{n}=\mathbf{4 2})\end{array}$ \\
\hline Arroz & 51 & 60 & 67 & 79 \\
Refrescos naturales & 40 & 28 & 39 & 38 \\
Frutas frescas & 37 & 53 & 33 & 21 \\
Vegetales & 29 & 23 & 22 & 15 \\
Cereales desayuno & 29 & 20 & 11 & 17 \\
Pan & 26 & 30 & 28 & 50 \\
Verduras harinosas & 26 & 8 & 11 & 7 \\
Frijoles & 20 & 35 & 39 & 48 \\
Avena, cebada, quinoa & 17 & 8 & 11 & 7 \\
Galletas saladas & 9 & 10 & 0 & 7 \\
Garbanzos, lentejas, arvejas & 9 & 3 & 0 & 2 \\
Salsa de tomate & 6 & 0 & 11 & 0 \\
Confites y dulces & 6 & 8 & 6 & 7 \\
Galletas dulces & 3 & 20 & 6 & 12 \\
Snacks & 3 & 0 & 6 & 2 \\
Spaguetti, Lasaña, Pastas & 3 & 0 & 6 & 0 \\
Granola & 3 & 10 & 0 & 0 \\
Tortillas & 3 & 8 & 0 & 0 \\
Repostería & 0 & 3 & 6 & 2 \\
Gaseosas & 0 & 0 & 6 & 5 \\
Pancakes, waffles & 0 & 0 & 6 & 0 \\
\hline
\end{tabular}

Tabla 5.

Consumo diario de proteína reportado por los participantes.

\begin{tabular}{lcccc}
\hline & \multicolumn{2}{c}{ Mujeres $(\mathbf{n}=\mathbf{7 5})$} & \multicolumn{2}{c}{ Hombres $(\mathbf{n}=\mathbf{6 0})$} \\
\cline { 2 - 5 } Consumo diario & $\begin{array}{c}\text { Modelo } \\
(\mathbf{n}=\mathbf{3 5})\end{array}$ & $\begin{array}{c}\text { Control } \\
(\mathbf{n}=\mathbf{4 0})\end{array}$ & $\begin{array}{c}\text { Modelo } \\
(\mathbf{n}=\mathbf{1 8})\end{array}$ & $\begin{array}{c}\text { Control } \\
(\mathbf{n}=\mathbf{4 2})\end{array}$ \\
\hline Leche & 49 & 28 & 39 & 55 \\
Huevos & 34 & 20 & 39 & 24 \\
Yogurt & 23 & 8 & 11 & 14 \\
Queso blanco & 14 & 15 & 17 & 14 \\
Carne de res & 11 & 3 & 6 & 7 \\
Pollo & 6 & 0 & 33 & 5 \\
Atún & 6 & 0 & 17 & 2 \\
Pescado & 3 & 0 & 28 & 5 \\
Mariscos & 3 & 0 & 0 & 0 \\
Carne de cerdo & 0 & 0 & 17 & 7 \\
Embutidos & 0 & 0 & 11 & 2 \\
Productos de soya & 0 & 3 & 0 & 5 \\
\hline
\end{tabular}

Nota: Los valores representan el porcentaje de respuestas en cada categoría.

Tabla 6 .

Consumo diario de grasas reportado por los participantes.

\begin{tabular}{lcccc}
\hline & \multicolumn{2}{c}{ Mujeres $(\mathbf{n}=\mathbf{7 5})$} & \multicolumn{2}{c}{ Hombres $(\mathbf{n}=\mathbf{6 0})$} \\
\cline { 2 - 5 } Consumo diario & $\begin{array}{c}\text { Modelo } \\
(\mathbf{n}=\mathbf{3 5})\end{array}$ & $\begin{array}{c}\text { Control } \\
(\mathbf{n}=\mathbf{4 0})\end{array}$ & $\begin{array}{c}\text { Modelo } \\
(\mathbf{n}=\mathbf{1 8})\end{array}$ & $\begin{array}{c}\text { Control } \\
(\mathbf{n}=\mathbf{4 2})\end{array}$ \\
\hline Aceite & 26 & 45 & 17 & 31 \\
Margarina/Mantequilla & 14 & 15 & 6 & 10 \\
Nueces: maní, almendras, etc. & 11 & & 17 & 2 \\
Aguacate & 9 & 3 & 6 & 2 \\
Mayonesa & 6 & & 6 & 2 \\
$\begin{array}{l}\text { Queso crema } \\
\text { Mantequilla de maní }\end{array}$ & 3 & & 6 & 5 \\
Helados & 3 & & 6 & \\
$\begin{array}{l}\text { Queso amarillo } \\
\text { Chocolates }\end{array}$ & 3 & & & 2 \\
Natilla & 0 & 3 & & \\
\hline
\end{tabular}

Nota: Los valores representan el porcentaje de respuestas en cada categoría

3). En el caso de la circunferencia de la cintura, se encontró que las mujeres modelos tenían una menor circunferencia de la cintura que las mujeres controles $(p<.001)$. Los hombres modelos y los controles tuvieron una similar circunferencia de la cintura $(p=.504)$ (Tabla 3). En el caso de la relación cintura/cadera, se encontró que las mujeres modelos tenían una menor relación cintura/cadera que las mujeres controles ( $p$ d» .001). Los hombres modelos y los controles tuvieron una similar circunferencia de la cintura ( $p=.862$ ) (Figura 1). Finalmente, en el caso del índice de conicidad, se encontró que las mujeres modelos tenían un menor índice de conicidad que las mujeres controles $(p<.001)$. Los hombres modelos y los controles tuvieron un índice de conicidad similar $(p=.692)$ (Figura 2).

La prueba t student para grupos independientes para la variable de tamaño del busto, reveló que las dimensiones eran similares entre las mujeres modelos (media $=82.25(5.33) \mathrm{cm}$ ) y las controles (media $=$ $84.95(6.67) \mathrm{cm})(p=.059)$.

Se realizó un análisis cualitativo de la alimentación de los participantes. La ingesta de alimentos reportada por los participantes fue variada. El consumo diario de alimentos fuentes de carbohidratos, proteínas y grasas se puede observar en las tablas 5, 6 y 7, respectivamente.

\section{Discusión}

En este estudio se encontró que el grado de adiposidad de los participantes se encontraba dentro de lo considerado como normal (American College of Sports Medicine, 2010, 2014). Sin embargo, $14.3 \%$ de las mujeres modelos y $2.5 \%$ de las mujeres controles se encontraban por debajo de $18.5 \mathrm{~kg} / \mathrm{m}^{2}$, considerado como bajo peso para su estatura. Esto ha sido criticado y actualmente en algunas pasarelas internacionales se ha llegado a acordar no permitir modelos con un IMC menor a $18.5 \mathrm{~kg} / \mathrm{m}^{2}$ (Stableford, 2012).

En este estudio también se encontró que los promedios de la relación cintura/cadera se encuentran dentro de lo considerado normal para hombres y para mujeres (American College of Sports Medicine, 2010, 2014). Finalmente, en cuanto al porcentaje de grasa corporal, los valores para las mujeres modelos están dentro del promedio, pero el de las mujeres control se encuentra sobre el promedio. Los índices de cintura/ cadera e IMC son indicadores generales asociados al grado de adiposidad de las personas; sin embargo, son técnicas correlacionadas con los denominados estándares de oro («gold standard»), como por ejemplo, DXA (Nana et al., 2015). En el presente estudio, la adiposidad fue medida con DXA, lo cual garantiza la alta validez de los datos obtenidos.

Los cambios en la idealización del cuerpo femenino se muestran en un estudio de finales de los años 90 (Morris et al., 1989), en el cual se contactó a una empresa de modelaje que tenía al menos 20 años de operar en este campo, y que contaba con información de modelos que participaron en revistas prestigiosas, de las cuales se tenía su record de las medidas de estatura, busto, cintura y cadera. Se examinaron 8 de los 20 años de los cuales se tenía la información completa. Se encontró que las modelos de modas estudiadas tendían a ser más altas, las medidas del busto y la cintura aumentaban con relación a las caderas, lo que modificaba la figura curvilínea a la forma corporal tubular(Morris et al., 1989). Se plantea que estos cambios en la forma corporal se hacen visibles en las revistas de moda y esto podría asociarse con el aumento en la prevalencia de dietas para alterar esta forma del cuerpo y desembocar en desórdenes alimenticios. Los autores de este estudio se apoyan en otras investigaciones relacionadas y en una de ellas se recolectó información tanto de una revista femenina como de las competidoras en un certamen de belleza durante un periodo de 20 años, de 1959 a 1978. Se encontró que a lo largo de ese tiempo ocurrió una disminución en el peso, y el promedio de la medida de la cintura aumentó, mientras que la de busto y cadera disminuyeron, lo que indicaba una tendencia a tener una forma corporal más tubular o andrógena (Morris et al., 1989). En el presente estudio se encontró que el índice de conicidad de las mujeres modelos era menor que el de las mujeres controles; es decir, tenían una forma corporal menos cilíndrica. En el caso de los hombres, tanto modelos como controles, la forma del cuerpo fue similar al evaluarla con el índice de conicidad.

En este estudio se encontró que la circunferencia del busto fue similar entre las mujeres modelos y las controles; es decir, tanto las modelos como las controles tuvieron un tamaño de busto similar, lo cual podría explicarse por el alto costo para realizarse cirugías cosméticas, porque ya no está de moda (al menos en Costa Rica) el aumento de senos por medio de implantes, o porque el mercado local no exige un busto de gran tamaño. En otras investigaciones (Furnham \& Swami, 2007; Treleaven, 2007) se ha determinado la contribución del tamaño del busto y de los glúteos al perfil del atractivo físico de las mujeres. Hay estudios (Bleske-Rechek, Kolb, Stern, Quigley, \& Nelson, 2014; Pawlowski \& Sorokowski, 2008), que se han enfocado en evaluar el atractivo físico basándose en el rostro, con la creencia de que es la zona que determina ese atractivo físico, pero los investigadores han redirigido su interés a estudiar los aspectos del atractivo físico, dentro del campo de la psicología evolutiva. Las características dominantes son la relación cintura cadera y IMC. Lo que ha pasado desapercibido son otras características del cuerpo como el busto y los glúteos. En un estudio en Inglaterra participaron 114 estudiantes de bachillerato que evaluaron 9 
siluetas con 3 niveles de tamaño de busto y de glúteos. Los resultados mostraron preferencia por el tamaño de busto pequeño, pero no con respecto al tamaño de los glúteos. Estos hallazgos sugieren que son atributos considerados menos importantes para el atractivo físico de las mujeres en el Reino Unido (Furnham \& Swami, 2007), a pesar de que una encuesta reveló que de 1920 a 2000 el busto de las mujeres británicas creció 10 cm y que pasaron de copa B a C (Treleaven, 2007).

Existe evidencia de tipo psicosocial que se enfoca en el impacto de la seguridad de los recursos (Dixson, Vasey, Sagata, Sibanda, Linklater, \& Dixson, 2011; Swami \& Tovee, 2013). Esta línea de investigación sugiere que el tamaño del busto puede actuar como una señal de reservas de energía (liberación de calor corporal) y alimento (leche), lo que indica un acceso a recursos. Con base en esto Swami y Tovee (2013), publicaron una investigación compuesta de dos estudios. En el primer estudio, participaron 266 hombres de 3 sitios de Malasia de diferentes estratos sociales, que evaluaron una serie de figuras que variaban en tamaño de busto.Los resultados mostraron que los hombres denivel socioeconómico bajo evaluaban el busto más grande como más atractivo que los de un contexto mediano, quienes percibían el busto también como atractivo, pero con los del nivel socioeconómico alto no sucedió lo mismo. El segundo estudio comparaba la relación del tamaño de busto en 66 hombres hambrientos comparados con 58 hombres saciados de Gran Bretaña. Los resultados indicaron que los hambrientos evaluaban más atractivos los bustos grandes en comparación con los hombres saciados. Estos estudios sugieren que el acceso de fuente, tiene un impacto en el juicio de ellos sobre la atracción de las mujeres (Swami \& Tovee, 2013). En otros países, el tamaño del busto puede ser una opción para obtener empleo y catapultarse al mundo del modelaje. Los cambios en el aspecto físico de una persona que está inmersa en la industria del modelaje pueden ser producto de la alimentación, al ejercicio o debido a otra técnica que se ha popularizado y que se ha vuelto muy importante para la industria de la cirugía cosmética (Draper, 1999).

En el presente estudio, se encontró que la relación cintura/cadera de las mujeres modelos era menor que las de las mujeres controles (.68 vs. .71), mientras que las de los hombres modelos fueron similares a las de los controles (1.23 vs. 1.22). En estudios previos (Brase \& Walker, 2004; Price, Pound, Dunn, Hopkins, \& Kang, 2013; Schmalt, 2006; Schützwohl, 2006), se plantean preferencias con respecto a las medidas que deben tener los modelos. Por ejemplo, las mediciones corporales que se asocian con el atractivo físico incluyen una relación cintura/cadera cercana a 7 (.68 a .72) en las mujeres (Brase \& Walker, 2004; Price, Pound, Dunn, Hopkins, \& Kang, 2013; Schmalt, 2006; Schützwohl, 2006), y en donde se define que la forma de pera es .75, la de reloj de arena es de .75, la de rectángulo es de .80 y de .82 la forma de manzana (Thoma et al., 2012).

Finalmente, se encontró que tres mujeres modelos presentaron escoliosis. Evidencia reciente(Fortin, Feldman, Cheriet, \& Labelle, 2013), indica que las personas que asumen posturas por largos periodos de tiempo (e.g., modelaje), pueden aumentar la progresión del desarrollo de la escoliosis, lo cual se convierte en un problema de salud para estas trabajadoras. Sin embargo, también se ha encontrado que una serie de ejercicios específicos realizados durante cuatro semanas podrían recudir la escoliosis (Maude, Head, \& Hobson, 2012) y mejorar la imagen corporal (Head, Maude, Black, Rolfe, \& Dorman, 2012), lo cual se convierte en una opción no quirúrgica importante si el grado de escoliosis (medida con el ángulo de Cobb) así lo permite. Se reconoce que este es un hallazgo incidental del presente estudio; que sin embargo, podría despertar el interés en estudiar las asociaciones entre el modelaje y el desarrollo de desórdenes en la postura.

De acuerdo a la información desplegada en la tabla 4, se puede observar que el arroz y los frijoles son los carbohidratos complejos más comunes en la dieta de los sujetos en general. La alta frecuencia de consumo de arroz y frijoles sigue el patrón de alimentación cultural de Costa Rica, donde ambos productos conforman hasta 30\% del consumo energético total por alimentos según encuestas nacionales de nutrición (Defensoría de los Habitantes y Consejo Nacional de Rectores,
2006; Mattei, Hu, \& Campos, 2011; Organización de las Naciones Unidas para la Alimentación y la Agricultura, 1999). El consumo de arroz duplica el de frijoles y es el único alimento reportado de las tablas 4-6 con una frecuencia de consumo superior al 50\% según el promedio total de todos los participantes. Una vez más, este patrón sigue las tendencias nacionales (Mattei et al., 2011; Ministerio de Salud de Costa Rica, 2001; Organización de las Naciones Unidas para laAlimentación y la Agricultura, 1999).

Las frutas frescas, los frescos naturales y el pan siguen al arroz y los frijoles como los carbohidratos consumidos con mayor frecuencia. El aumento en el consumo de carbohidratos refinados como el pan ha venido en aumento en nuestra región, al mismo tiempo que se ha reducido el consumo de frutas y vegetales (Bermudez \& Tucker, 2003; De Piero, Bassett, Rossi, \& Samman, 2015). No obstante, todavía se mantiene un consumo regular considerable de frutas frescas y frescos naturales que no obedece únicamente a los modelos, sino a ambos grupos según la tabla 4, siguiendo nuevamente un perfil nutricional autóctono (Organización de las Naciones Unidas para laAlimentación y laAgricultura, 1999).

El modelaje tiene como «requisito» cierto aspecto físico que conlleva, en la mayoría de casos, a una rutina de ejercicios específica de gimnasio combinada con una dieta controlada, creando una necesidad de educarse individualmente o buscar ayuda profesional en el tema. Un manejo de conceptos nutricionales por parte de los modelos se observa por una menor frecuencia de consumo de arroz en relación a los participantes controles, unido a un mayor consumo de verduras harinosas y cereales integrales en su lugar (e.g., avena, cebada, quínoa, polenta); preferencia por grasas saludables (e.g., nueces, aguacate) y menor consumo de galletas dulces, repostería y aceite vegetal.

Siguiendo con la idea anterior de una alimentación dirigida a un aspecto físico para el modelaje, la característica más esperable en la dieta es una alta frecuencia de consumo de proteína, confirmado en la tabla 5; los modelos, sin importar sexo, reportan un consumo más frecuente que el control de huevo y carnes en general. Dietas con cantidades de proteína superiores a las cantidades diarias recomendadas (o RDA por sus siglas en inglés $=0.8 \mathrm{~g} / \mathrm{kg}$ ) son una constante en el mundo del modelaje al ser asociadas culturalmente con un mejor aspecto corporal al fomentar el desarrollo de masa muscular, controlar el peso y proteger la masa magra en restricciones energéticas (Helms, Aragon, \& Fitschen, 2014; Helms, Zinn, Rowlands, \& Brown, 2014; Murphy, Hector, \& Phillips, 2015; Pasiakos et al., 2013; Phillips, 2014). En relación a los diferentes tipos de carne, el pollo y el pescado son los que se consumen con mayor frecuencia, lo que refuerza cierto dominio de conceptos al ser las carnes más bajas en grasa.

La diferencia entre géneros en el consumo de proteína ha sido previamente documentada (De Piero et al., 2015). Los diferentes tipos de modelaje masculinos exigen una marcada musculatura, que puede verse asociada entonces con mayor consumo proteico; mientras que los diferentes tipos de modelaje femenino no necesariamente demandan un gran desarrollo muscular. En el caso de las mujeres modelos, las dietas altas en proteína pueden verse más asociadas a un desplazamiento de los carbohidratos buscando una disminución del peso.

La mayor frecuencia de consumo de grasas (especialmente saturadas) y de líquido (frescos naturales y gaseosas) por parte de los hombres en comparación a las mujeres sin importar el grupo también siguen patrones de género estudiados (De Piero et al., 2015; Leblanc, Begin, Corneau, Dodin, \& Lemieux, 2015; Rhee, Mattei, \& Campos, 2012). En general, las mujeres tienden a ser más cautas que los hombres en cuanto a su patrón de alimentación (Leblanc et al., 2015), de ahí que busquen evitar estrictamente grasas y alimentos azucarados por su alto contenido energético, baja cantidad de nutrientes y por estar ligados a efectos que podrían perjudicar su apariencia física.

El presente estudio reconoce varias limitaciones y delimitaciones. Primero, el estudio está delimitado a la población costarricense de modelos y personas controles; por lo que los hallazgos no se pueden generalizar a otras poblaciones. Segundo, podría haber un sesgo de 
selección, pues modelos costarricenses que participan en pasarelas fuera del país podrían no representar a las y los modelos que participaron en el presente estudio. Sin embargo, se realizaron intentos para reducir este sesgo, como por ejemplo, tratar de reclutar participantes de múltiples agencias de modelaje de todo el país, ir a eventos de pasarelas a reclutar participantes personalmente, e incluso, comunicarse con modelos de reconocido renombre por medio de sus representantes o sus perfiles en redes sociales (e.g. Facebook).

En conclusión, el IMC de las y los modelos, así como de los controles, estaba dentro de lo considerado normal. Una pequeña proporción de mujeres modelos (14.3\%) y controles (2.4\%) se encontraba con un bajo peso para su estatura (i.e., IMC $<18.5 \mathrm{~kg} / \mathrm{m}^{2}$ ). Sin embargo, el porcentaje de grasa corporal de las mujeres control se encuentra sobre el promedio. La forma corporal de las modelos era menos cilíndrica que la de las mujeres controles; mientras que las de los hombres eran similares. La circunferencia del busto fue similar entre las mujeres modelos y las controles, y se encontró que tres modelos mujeres presentaron escoliosis. Los patrones de alimentación son similares entre los participantes. El arroz y los frijoles son los carbohidratos complejos más comunes en la dieta. Los modelos manejan conceptos nutricionales apropiados, como por ejemplo, una menor frecuencia de consumo de arroz en relación a los controles, unido a un mayor consumo de verduras harinosas y cereales integrales; preferencia por grasas saludables y menor consumo de galletas dulces, repostería y aceite vegetal. Además, los modelos, sin importar sexo, reportan un consumo más frecuente de proteínas que los controles.

\section{Referencias}

American College of Sports Medicine. (2010). ACSM's health-related physical fitness assessment manual ( $3^{\text {rd }}$ ed.). Philadelphia, PA.: Wolters Kluwer, Lippincott Williams \& Wilkins.

American College of Sports Medicine. (2014). ACSM's Guidelines for Exercise Testing and Prescription ( $9^{\text {th }}$ ed.). Philadelphia, PA.: Wolters Kluwer, Lippincott Williams \& Wilkins.

Bermudez, O. I., \& Tucker, K. L. (2003). Trends in dietary patterns of Latin American populations. Cadernos de Saúde Pública, 19, S87-S99.

Bleske-Rechek, A., Kolb, C. M., Stern, A. S., Quigley, K., \& Nelson, L. A. (2014). Face and body: independent predictors of women's attractiveness. Arch Sex Behav, 43(7), 1355-1365. doi:10.1007/s10508-014-0304-4

Brase, G. L., \& Walker, G. (2004). Male sexual strategies modify ratings of female models with specific waist-to-hip ratios. Human Nature, 15(2), 209-224.

Carbuhn, A. F., Fernandez, T. E., Bragg, A. F., Green, J. S., \& Crouse, S. F. (2010). Sport and training influence bone and body composition in women collegiate athletes. $J$ Strength Cond Res, 24(7), 1710-1717. doi:10.1519/JSC.0b013e3181d09eb3

De Piero, A., Bassett, N., Rossi, A., \& Samman, N. (2015). [Trends in food consumption of university students]. Nutr Hosp, 31(4), 1824-1831. doi:10.3305 nh.2015.31.4.8361

Defensoría de los Habitantes y Consejo Nacional de Rectores. (2006). Duodécimo informe Estado de la Nación en Desarrollo Humano Sostenible. Principales tendencias de la situación nutricional de la población de Costa Rica y su atención en el periodo 1996-2006. San José, Costa Rica: Gobierno de Costa Rica.

Dixson, B. J., Vasey, P. L., Sagata, K., Sibanda, N., Linklater, W. L., \& Dixson, A. F. (2011) Men's preferences for women's breast morphology in New Zealand, Samoa, and Papua New Guinea. Arch Sex Behav, 40(6), 1271-1279. doi:10.1007/s10508010-9680-6

Draper, A. E. (1999). 'I'm sorry, you said she was how old?’Youthfulness and the fashion model. Social Alternatives, 18(2), 33-37.

Fernandez-Campos, C., Dengo, A. L., \& Moncada-Jimenez, J. (2015). Acute consumption of an energy drink does not improve physical performance of female volleyball players. Int J Sport Nutr Exerc Metab, 25, 271-277. doi:10.1123/ijsnem.2014-0101

Fortin, C., Feldman, D. E., Cheriet, F., \& Labelle, H. (2013). Differences in Standing and Sitting Postures of Youth with Idiopathic Scoliosis from Quantitative Analysis of Digital Photographs. Physical \& Occupational Therapy in Pediatrics, 33(3), 313-326. doi:10.3109/01942638.2012.747582

Furnham, A., \& Swami, V. (2007). Perception of female buttocks and breast size in profile. Social Behavior \& Personality, 35(1), 1-7.

Head, J., Maude, E., Black, J., Rolfe, T, \& Dorman, R. (2012). Does a four-week intensive scoliosis-specific exercises programme improve body-image in subjects with idiopathic scoliosis and is the effect rated equally by patients, physiotherapists and an external rater with scoliosis? Scoliosis, 7(1), 1-1. doi:10.1186/1748-7161-7s1-052

Helms, E. R., Aragon, A. A., \& Fitschen, P. J. (2014). Evidence-based recommendations for natural bodybuilding contest preparation: nutrition and supplementation. J Int Soc Sports Nutr, 11, 20. doi:10.1186/1550-2783-11-20

Helms, E. R., Zinn, C., Rowlands, D. S., \& Brown, S. R. (2014). A systematic review of dietary protein during caloric restriction in resistance trained lean athletes: a case for higher intakes. Int J Sport Nutr Exerc Metab, 24(2), 127-138. doi:10.1123/ ijsnem.2013-0054

Ito, M., Nakamura, T., Ikeda, S., Tahara, Y., Hashmi, R., Tsurusaki, K., ...Hayashi, K. (2001) Effects of lifetime volleyball exercise on bone mineral densities in lumbar spine, calcaneus and tibia for pre-, peri- and postmenopausal women. Osteoporos Int, 12(2), 104-111. doi:10.1007/s001980170141

Leblanc, V., Begin, C., Corneau, L., Dodin, S., \& Lemieux, S. (2015). Gender differences in dietary intakes: what is the contribution of motivational variables? J Hum Nutr Diet, 28(1), 37-46. doi:10.1111/jhn.12213

Mattei, J., Hu, F. B., \& Campos, H. (2011).A Ahigher ratio of beans to white rice is associated with lower cardiometabolic risk factors in Costa Rican adults. Am J Clin Nutr, 94(3), 869-876. doi:10.3945/ajcn.111.013219

Maude, E., Head, J., \& Hobson, K. (2012). The effect of a four-week intensive scoliosisspecific exercises programme on Cobb angle in subjects with idiopathic scoliosis: an 11 patient case series. Scoliosis, 7(1), 1-1. doi:10.1186/1748-7161-7-s1-o51

Ministerio de Salud de Costa Rica. (2001). Encuesta nacional de consumo de alimentos. Encuesta de hogares y de propósitos múltiples. San José, Costa Rica: Ministerio de Salud.

Moncada-Jiménez, J. (2006, 02/10/06). Madrid y la salud de las modelos. La Nación, pp. 44-A. Retrieved from http://www.nacion.com/opinion/Madrid-saludmodelos_0_858314222.html

Montero, I., \& León, O. G. (2002). Clasificación y descripción de las metodologías de investigación en Psicología. International Journal of Clinical and Health Psychology, 2(3), 503-508.

Morris, A., Cooper, T., \& Cooper, P. J.(1989). The changing shape of female fashion models International Journal of Eating Disorders, 8(5), 593-596. doi:10.1002/1098108x(198909)8:5<593::aid-eat2260080511>3.0.co;2-h

Murphy, C. H., Hector, A. J., \& Phillips, S. M. (2015). Considerations for protein intake in managing weight loss in athletes. Eur J Sport Sci, 15(1), 21-28. doi:10.1080/ 17461391.2014.936325

Nana, A., Slater, G. J., Stewart, A. D., \& Burke, L. M.(2015). Methodology review: using dual-energy X-ray absorptiometry (DXA) for the assessment of body composition in athletes and active people. Int J Sport Nutr Exerc Metab, 25(2), 198-215. doi:10.1123/ijsnem.2013-0228

Organización de las Naciones Unidas para la Alimentación y la Agricultura. (1999). Perfiles nutricionales por países: Costa Rica. Retrieved from San José, Costa Rica: $\mathrm{ftp}: / / \mathrm{ftp}$. fao.org/ag/agn/nutrition/ncp/crimap.pdf

Pasiakos, S. M., Cao, J. J., Margolis, L. M., Sauter, E. R., Whigham, L. D., McClung, J. P., .. Young, A. J. (2013). Effects of high-protein diets on fat-free mass and muscle protein synthesis following weight loss: a randomized controlled trial. FASEB $J$, 27(9), 3837-3847. doi:10.1096/fj.13-230227

Pawlowski, B., \& Sorokowski, P. (2008). Men's attraction to women's bodies changes seasonally. Perception, 37(7), 1079-1085.

Phillips, S. M. (2014). A brief review of higher dietary protein diets in weight loss: a focus on athletes. Sports Med, 44 Suppl 2, S149-153. doi:10.1007/s40279-014-0254-

Preti,A., Usai, A., Miotto, P., Petretto, D. R., \& Masala, C. (2008). Eating disorders among professional fashion models. Psychiatry Research, 159(1-2), 86-94. doi:10.1016/ j.psychres.2005.07.040

Price, M. E., Pound, N., Dunn, J., Hopkins, S., \& Kang, J. (2013). Body shape preferences: associations with rater body shape and sociosexuality. PLoS One, 8(1), e52532. doi:10.1371/journal.pone.0052532

Rhee, J. J., Mattei, J., \& Campos, H. (2012). Association between commercial and traditiona sugar-sweetened beverages and measures of adiposity in Costa Rica. Public Health Nutr, 15(8), 1347-1354. doi:10.1017/s1368980012001000

Santonastaso, P., Mondini, S., \& Favaro, A. (2002). Are fashion models a group at risk for eating disorders and substance abuse? Psychotherapy and Psychosomatics, 71(3), 168-172, doi:10.1159/000056285

Schmalt, H.-D. (2006). Waist-to-hip ratio and female physical attractiveness: The moderating role of power motivation and the mating context. Personality and Individual Differences, 41(3), 455-465. doi:http://dx.doi.org/10.1016/ j.paid.2006.02.008

Schützwohl, A. (2006). Judging female figures: A new methodological approach to male attractiveness judgments of female waist-to-hip ratio. Biological Psychology, 71(2), 223-229. doi:http://dx.doi.org/10.1016/j.biopsycho.2005.04.005

Stableford, D. (2012). Israel's skinny model ban: New law requires body mass index of 18.5 or a doctor's note. Retrieved from http://news.yahoo.com/blogs/cutline/ israel-skinny-model-ban-law-requires-body-mass-160408015.html

Swami, V., \& Tovee, M. J. (2013). Resource security impacts men's female breast size preferences. PLoS One, 8(3), e57623. doi:10.1371/journal.pone.0057623

Sypeck, M. F., Gray, J. J., \& Ahrens, A. H. (2004). No longer just a pretty face: Fashion magazines' depictions of ideal female beauty from 1959 to 1999. International Journal of Eating Disorders, 36(3), 342-347. doi:10.1002/eat.20039

Thoma, M.E., Hediger, M. L., Sundaram, R., Stanford, J. B., Peterson, C. M., Croughan, M. S., ... Buck Louis, G. M. (2012). Comparing apples and pears: women's perceptions of their body size and shape. J Womens Health (Larchmt), 21(10), 1074-1081. doi:10.1089/jwh.2012.3634

Treleaven, P. (2007). How to fit into your clothes. Significance, 4(3), 113-117. doi:10.1111/j.1740-9713.2007.00243.x

Valdez, R., Seidell, J. C., Ahn, Y. I., \& Weiss, K. M. (1993). A new index of abdominal adiposity as an indicator of risk for cardiovascular disease. A cross-population study. Int J Obes Relat Metab Disord, 17(2), 77-82.

Valdimarsson, O., Alborg, H. G., Duppe, H., Nyquist, F., \& Karlsson, M. (2005). Reduced training is associated with increased loss of BMD. J Bone Miner Res, 20(6), 906912. doi:10.1359/jbmr.050107 